\title{
Hybrid consumption paths in the attribute space: A model and application with scanner data.
}

\author{
Sergi Jiménez-Martín* Antonio Ladron-de-Guevara Martinez ${ }^{\dagger}$
}

This version: February 2008.

\begin{abstract}
Abstract: This paper presents and estimates a dynamic choice model in the attribute space considering rational consumers. In light of the evidence of several state-dependence patterns, the standard attribute-based model is extended by considering a general utility function where pure inertia and pure variety-seeking behaviors can be explained in the model as particular linear cases. The dynamics of the model are fully characterized by standard dynamic programming techniques. The model presents a stationary consumption pattern that can be inertial, where the consumer only buys one product, or a variety-seeking one, where the consumer shifts among varied products.

We run some simulations to analyze the consumption paths out of the steady state. Under the hybrid utility assumption, the consumer behaves inertially among the unfamiliar brands for several periods, eventually switching to a variety-seeking behavior when the stationary levels are approached. An empirical analysis is run using scanner databases for three different product categories: fabric softener, saltine cracker, and catsup. Non-linear specifications provide the best fit of the data, as hybrid functional forms are found in all the product categories for most attributes and segments. These results reveal the statistical superiority of the non-linear structure and confirm the gradual trend to seek variety as the level of familiarity with the purchased items increases.
\end{abstract}

\footnotetext{
${ }^{*}$ Universitat Pompeu Fabra and FEDEA, Department of Business and Economics, C/Ramon Trias Fargas 25-27, 08004 Barcelona, Spain. E-mail: sergi.jimenez@upf.edu

${ }^{\dagger}$ Department of Business and Economics, Universitat Pompeu Fabra, C/Ramon Trias Fargas 25-27, 08004 Barcelona, Spain. E-mail: antonio.ladron@upf.edu
} 


\section{Introduction:}

In the last decades, the vast empirical evidence on purchase probabilities being affected by the previously purchased history has fostered relevant lines of research on consumer state-dependence models. However, the observed direction of the effect varies depending on the market characteristics and the product category. In some situations the purchase of an item by a consumer decreases the probability that it will be purchased on the next occasion. This pattern is known in the literature as a "variety-seeking" (see McAlister 1982, Givon 1984, Lattin and McAlister 1985, and Kahn, Kalwani and Morrison 1986). On the contrary, the variety-avoiding behavior is present when the choice of an item leads to an increase in the probability of selecting it on a subsequent choice occasion, and the implied pattern is said to be inertial (see Kuehn 1962, Morrison 1966, Jeuland 1979, Guadagni and Little 1983, Givon 1984, and Tversky and Kahneman 1991, Hardie, Johnson and Fader 1993, and Fader and Hardie 1996)

Most of the referred models include information on last purchased brand (1st-order brand models) to explain present decisions. However, later research on state dependence adopts two relevant extensions. First, some authors consider additional attributes besides brand to explain purchase decisions. Keane (1997) includes dummy variables to capture preferences on brands, plastic containers and sizes. Kim, Allenby and Rossi (2002) include flavor in the yogurt market and find evidence of simultaneous demand for varied flavors while there is virtually no brand switching between the two leading brands. The second line of extension follows the methodology proposed by McAlister (1982) and Guadagni and Little (1983), and considers a higher order state-dependence level as the utility derived from goods is based on the cumulative levels of attributes. This approach uses exponentially smoothed measures of previous purchases to better capture the past consumption history and proposes utility functions determined by the cumulative level of the compounding attributes (see Lattin 1987, Fader and Hardie 1996, and Jimenez-Martin and Ladrón-de-Guevara 2007).

Linear specifications for the state-dependence partial utilities do not allow for a behavior where both inertia and variety seeking may coexist within the individual for the same attribute. However, some empirical evidence consistent with a mixed behavior was originally been reported by Wierenga (1974), who observed that consumers tend to fluctuate between repeat purchasing and brand-switching behavior for frequently purchased products. From now on, we refer to this mixture of inertia and variety seeking as "hybrid" behavior. Some theoretical framework in the psychology literature accounts for a hybrid behavior: Berlyne $(1963,1970)$ proposes that the 
attractiveness of a stimulus is an inverted-U shaped function of its level of familiarity. According to this theory, an inertial behavior comes into play when the individual is exposed to a relatively unfamiliar stimulus and there is a tendency to repeat it, increasing the level of familiarity. In fact, the ability to enjoy new attributes is a process that usually requires successive consumption of the same item: It takes time and several trials for a consumer to get used to new musical styles or to develop the ability to appreciate wines. High familiarity, on the other hand, brings the variety-seeking pattern into play, leading to an increasing tendency to look for other stimuli. According to this theory, if repeat purchasing of an item leads to greater familiarity with it, the consumer will switch from an inertial to a variety-seeking pattern once a certain number of repeat purchases are made. This hybrid behavior may be explained assuming inverted-U partial utility functions. Bawa (1990) finds evidence of this concave structure using panel datasets for the facial tissue and paper towel categories at a household level. ${ }^{1}$ Jimenez-Martin and Ladrón de Guevara (2007) also report relevant segments presenting this hybrid behavior for several product categories.

Noteworthy, contribution in this area has been only empirical, as none of the referred research studies the dynamics and the implied optimal consumption patterns derived from the statedependence structure assuming rational consumers. State-dependence assumptions imply that present decisions will affect future utilities. This intertemporal interaction between present consumption, attribute accumulation, and future utility requires a dynamic framework to be modeled. Investment is an intertemporal concept and can be defined as the employment of resources in the acquisition of anything from which a future profit is expected. This concept has extensively been used in all sorts of economic models to study both firm and household optimal decisions (e.g. research and development, advertising, household production, economic growth, etc.). As long as future tastes are continuously modified by the stock of attributes accumulated by the past consumption history, a rational agent will plan the consumption pattern across several periods as an attribute-investment process.

This article presents and estimates a dynamic choice model in the attribute space. We extend the empirical framework proposed by authors like McAlister (1982), Lattin (1987), Bawa (1990), Fader et al. (1996), and Jimenez-Martin et. al.(2007), by considering an economic consumerchoice model with rational consumers that discount the future. All the previous research on

\footnotetext{
${ }^{1}$ Considering the brand as the only valuable attribute, Bawa finds evidence of hybrid behavior using panel datasets at a household level. However, the model assumes that each time a brand switch occurs, the choice process "renews", so the state-dependence assumption is only valid for the last purchases after the last switch. This assumption seems to be too restrictive for categories where consumers constantly seek variety.
} 
consumer choice to date assume myopic agents that do not discount future utilities when taking present decisions.

In light of the evidence of several state-dependence patterns, we relax the linearity assumption by considering a general utility function Once the dynamic model is stated, the resulting maximization problem is solved analytically. We determine the first-order conditions and characterize the optimal consumption paths. The model presents a stationary consumption pattern that can be inertial, where the consumer only buys one product, or a variety-seeking one, where the consumer buys several products simultaneously. We run some simulations to determine the transitional optimal consumption plan since an agent tries a new product category, departing from a zero stock level of attributes, until reaching the steady-state equilibrium. Under the inverted-U marginal utility assumption (Bawa 1990 and Jimenez-Martin et al 2007) the consumer behaves inertially among the existing brands for several periods, and eventually, once the stationary stock levels are approached, the consumer may turn to a variety-seeking behavior.

After the analysis of the dynamics of the model, we run an empirical exercise to estimate the marginal utility functions for the several compounding attributes using three well known scanner databases for nondurable indivisible goods: fabric softeners, saltine crackers, and catsup. The datasets have been previously used by several authors: Jain, Vilcassim, and Chintagunta (1994), Roy, Chintagunta, and Haldar (1996), Fader et al.(1996), and Jiménez-Martín et al. (2007). Heterogeneity is introduced in the estimation process by allowing for multiple latent consumption segments. At the attribute level, empirical evidence of hybrid behavior is found for most attributes in all three markets

The outline of the paper is as follows. In section 2 the model is introduced, along with a basic discussion of the long-run optimal stationary path, and the main analytical results for the characterization of both inertial and variety-seeking long-run patterns are presented. In section 3 we analyze the consumption patterns for new categories or new attributes, assuming a utility functional form that allows for a hybrid behavior. In section 4 the indivisible-good version of the model with categorical attributes is developed, to account for more realistic choice and consumption processes. In section 5 an application of the model with indivisible goods and categorical attributes is presented. The conclusions are presented in section 6 with some managerial implications of the results and proposals for further developments and extensions of the model. 


\section{The General Model}

The general model developed in this section describes the optimal consumption path in a dynamic framework. The objective is to model the state-dependence consumption pattern within a frequent-purchase category, in a manner that allows for the different types of behavior described in the literature: pure inertia, pure variety seeking, and hybrid.

Consistent with the characteristics models [see Lancaster (1971)] the approach followed relates the preference for a product to the preference contributions of the attributes derived from its consumption. In line with models like the ones proposed by McAlister (1982) and McAlister and Pessemier (1982), the utility in each consumption period is derived from the attribute inventories accumulated when an item is consumed. The attribute inventory is hypothesized to depreciate continuously, and experiences discrete increments each period an item containing this attribute is consumed. The cumulative stock for the attribute $j$ in period $t$, say $s_{k a t}(t)$, is determined by the following law of motion:

$$
\operatorname{stkat}_{j}(t)=i n v_{j}(t)+\left(1-\lambda_{j}\right) \operatorname{stkat} t_{j}(t-1)
$$

where $i n v_{j}(t)$ is the amount of attribute $j$ derived from consumption of an item in period $t$, and $\lambda_{j}$ is the corresponding depreciation rate.

As proposed by Lancaster (1971), when an item is consumed, the contribution to each attribute is assumed to be determined by the following linear consumption technology:

$$
i n v_{j}(t)=\sum_{i=1}^{I} b_{j i} q_{i}(t)
$$

where $q_{i}(t)$ is the quantity of good $i$ consumed at period $t$ and $b_{j i}$ is the quantity of the characteristic $j$ contained in one unit of good $i$. Noteworthy, if there were two homogeneous goods (identical proportions of the constituent characteristics) the agent would only consume the efficient one on the basis of cost, showing no demand for the other good. Then, without loss of generality, from now on it will be assumed that all the available goods are differentiated.

The consumer is a utility maximizer, responding to a local temporal budget constraint. In line with Thaler (1985), the budgeting process is assumed to occur on a periodical basis for each category. Given the prices and category-specific budget constraint, the consumer evaluates purchases as situations arise. Assuming a previously determined budget for every period, say 
$m(t)$, and a vector of exogenous prices $p(t)$, the consumer will face the following restriction:

$$
\sum_{i=1}^{I} p_{i}(t) q_{i}(t) \leq m(t)
$$

In line with the attribute-based theory, every period $t$ consumers are assumed to derive utility from the cumulative stock levels of attributes, captured by the $J$-dimensional variable stkat $(t)$, when consuming a good. The one-period utility, $u(\operatorname{stkat}(t))$, is assumed to increase monotonically for all the attributes.

In the described framework, the decisions on present consumption affect the future utilities through the depreciated stock of attributes. Assuming that the future utility is discounted at a rate $\delta$, a rational consumer decides the optimal consumption path by maximizing the discounted flow of utilities derived from the attribute levels reached in every period. Given the initial stock of attributes, stkat $t_{0}$, the optimization problem for the consumer consists of choosing the quantities of goods $q_{i}$ to be bought at every period $t$, subject to the budget restriction (3), to increase the inventory levels of the compounding attributes stkat according to the socking process determined by equations (1) and (2), in order to maximize the discounted flow of utilities:

$$
\max _{\left\{q_{1}(t), q_{2}(t), \ldots, q_{I}(t)\right\}_{t=1}^{\infty}} \sum_{t=1}^{\infty}(1-\delta)^{t} u[\operatorname{stkat}(t)]
$$

subject to:

$$
\begin{gathered}
\sum_{i=1}^{I} p_{i}(t) q_{i}(t) \leq m(t) \quad \forall t \\
\operatorname{inv}_{j}(t)=\sum_{i=1}^{I} b_{j i} q_{i}(t) \quad \forall j, \forall t \\
\operatorname{stkat}_{j}(t)=\operatorname{inv}_{j}(t)+\left(1-\lambda_{j}\right) \operatorname{stkat}(t-1) \quad \forall j, \forall t \\
\operatorname{stkat}_{j}(0)=\operatorname{stkat}_{j 0} \quad \forall j
\end{gathered}
$$

\subsection{The Optimal Consumption Pattern in the attribute space}

In order to analyze the dynamics of the model, it is convenient to characterize the optimal solution of the problem stated in the previous section, in the attribute space, stkat. Every combination of goods $q(t)$ consumed in period $t$ contribute to increase the stocks of attributes $s t k a t(t)$ from which the utility is derived. In the attribute-based framework, the economy may 
be simple or complex, depending on the number of available goods compared to the constituent attributes. As defined by Lancaster (1971), in a complex economy the number of available products, $I$, exceeds the number of attributes, $J$. In this case, every attribute vector can be achieved by infinite combinations of goods, but the optimizing consumer will limit his choice to the subset of goods that constitute the attribute frontier, known as the efficient goods. This gives a one-to-one relationship between the sub-set of efficient goods and the attributes with an implied zero demand for the remaining $I-J$ goods. Figure 1a illustrates a complex economy constituted by four available goods, represented as vectors in the 2-dimensional attribute space, where only combinations of the goods 1 and 2 allow for efficient allocations.

On the other side, if the market is characterized by a wide range of characteristics, the opposite case may be presented, with more attributes than available brands $(J>I)$. Some products like automobiles are made up of a great amount of constituent features and attributes, in what Lancaster defines as a simple economy. The economy is simple in the sense that the number of available items do not allow for acquiring every combination of attributes, so the consumer is limited to the available subset of implied combinations. In a simple economy the consumer will be limited to choose in the subspace of dimension $I$ generated by the available set of items. The optimization problem can be solved by considering the reduced system of any arbitrarily chosen subset of $I$ attributes, with the remaining $J-I$ attributes being implicitly determined. Figure 1b illustrates a simple economy with only two available goods in a three dimensional attribute space. In this case, the consumer maximizes the utility in the two-dimensional subspace determined by combinations of both goods. ${ }^{2}$

Complex Economy 4 goods, 2 attributes

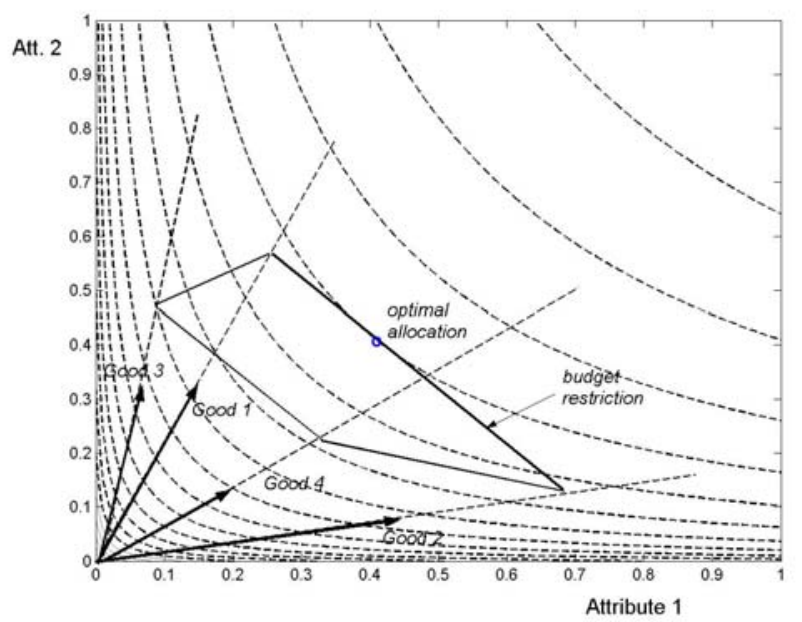

Simple Economy

2 goods, 3 attributes

\footnotetext{
${ }^{2}$ See Lancaster (1971) for a detailed analysis of the efficient choice set in the attribute space.
} 
Figures 1a and 1b: Illustration of complex and simple economies in the attribute space.

In both the simple and the complex economies, the number of efficient goods equals the number of linearly independent attributes, so there is a one-to-one relationship between every possible combination of goods and the implied combination of attributes. The square matrix $B$ of the reduced system can be inverted and without loss of generality the maximization problem can be expressed in the attribute space. The one-period budget restriction (3) can then be expressed in terms of the level of attributes derived from consumption of the goods purchased:

$$
\sum_{j=1}^{J} p_{j}^{\prime}(t) i n v_{j}(t) \leq m(t) \quad \forall t
$$

where the price of acquiring one unit of attribute $j$ at time $t$, denoted by $p_{j}^{\prime}(t)$, is a linear function of the good-price vector $p(t)$ and the inverse of the technology matrix:

$$
p_{j}^{\prime}(t)=\sum_{i=1}^{I} b_{i j}^{-1} p_{i}(t)
$$

To characterize the optimal solution of problem $(\mathrm{P})$, we consider a continuous, strictly increasing, twice differentiable, and concave utility function, $u($ stkat), and a compact and convex set defined by the constraint equation system (1)-(3). Under these assumptions, the following first-order conditions characterize the interior solution when nonsatiation of the attributes is also assumed. By using equations (4) and (5), the first-order conditions can be expressed in the attribute space:

$$
\begin{aligned}
& \sum_{j=1}^{J} p_{j}^{\prime}(t) \operatorname{stkat}_{j}(t)=m(t)+\sum_{j=1}^{J} p_{j}^{\prime}(t)\left(1-\lambda_{j}\right) \operatorname{stkat}_{j}(t-1) \quad t=1,2, \ldots \\
& \frac{p_{v}^{\prime}(t)}{p_{w}^{\prime}(t)}=\frac{\sum_{n=0}^{\infty}(1-\delta)^{n}\left(1-\lambda_{v}\right)^{n} u_{v}[\operatorname{stkat}(t+n)]}{\sum_{n=0}^{\infty}(1-\delta)^{n}\left(1-\lambda_{w}\right)^{n} u_{w}[\operatorname{stkat}(t+n)]}, \quad \forall v, w \in J \quad t=1,2, \ldots
\end{aligned}
$$

The first equation is the budget restriction expressed in terms of the attribute inventories stkat $(t)$ and defines the maximum stocks attainable given the price vector $p(t)$, the budget $m(t)$, and the depreciated stock-of-attribute vector from the previous period stkat $(t-1)$. This is a first-order difference equation, so at every period, the attribute frontier is a function of the previous-period optimal stocks of attributes. The second equation characterizes interior solutions where strictly positive quantities of all goods are consumed. For interior solutions the 
price ratio between every two attributes equals the ratio between the discounted flows of future marginal utilities derived from an additional unit of attribute. When interiority is presented the consumer behaves variety-seeking as all the durable goods are consumed simultaneously at every period. However, the model may also present non-interior solutions where only one good is consumed along the optimal path. When products are close substitutes the optimal consumption pattern may not be interior, implying an inertial behavior, even under strongly concave preferences. ${ }^{3}$

Assuming that in a frequent-consumption category the agent expects the price vector and the budget to be constant for several periods, the consumption path approaches a stationary pattern. If the price vector $p(t)$ and the budget $m(t)$ remain constant, the restriction (6) will monotonically converge to a long-run budget restriction, where in every period the consumer faces the same attribute frontier. Once the stationary restriction is reached, the budget for every period is completely used to restore the depreciated levels of attributes. If this is the case, the consumption path has reached a stationary pattern where the purchased quantities and the attribute stocks remain constant period by period. In the long run the consumer splits the budget to restore the depreciated stocks of attributes, maintaining an optimal proportion among the different attributes.

As in every standard concave dynamic problem, the steady state governs the transitional dynamics, so any optimal path out of the steady state from any initial conditions is driven by the convergence dynamics towards it. If there is a change in prices or any other variable assumed to be constant, the long-run frontier will shift and the problem will present a new steady state, but the consumption pattern will again be governed by the convergence dynamics, eventually approaching the new steady-state values in the long run. This is the main rationale and motivation to study the stationary consumption path.

Definition 2.1: For a given constant budget restriction, $m$ and a constant price vector, $p$, a stationary consumption path is defined as a consumption sequence, $\{q(t), t=1,2, \ldots\}$ that solves the optimization problem (P) for a certain initial condition, stkat $t_{0}$, such that the resulting vector of attribute stocks along the path, $\{\operatorname{stkat}(t), t=1,2, \ldots\}$ remains fixed over time.

From now on, without loss of generality, our analysis will be limited to the case where consumers derive utility from two different attributes and two items within a certain category. The

\footnotetext{
${ }^{3}$ A utility function is said to be strongly concave when the marginal utility approaches infinity as the related attribute goes to 0 . As a result, the indifference curves never cross the axes, so a strictly positive level of every attribute is needed to derive utility.
} 
following results and conclusions can be extended to a higher-dimension problem. Let's assume that the exogenous budget $m(t)$ and the price vector $p(t)$ remain constant, so a convergence process towards a stationary pattern is presented. The optimal consumption path characterized by the first order conditions will eventually converge to a steady-state consumption path. Imposing stationarity in the budget restriction (6) and in the first-order condition (7), the following equation system is obtained:

$$
\begin{aligned}
m & =\lambda_{1} p_{1}^{\prime} s t k a t_{1}^{*}+\lambda_{2} p_{2}^{\prime} \text { stkat } t_{2}^{*} \\
\frac{p_{1}^{\prime}}{p_{2}^{\prime}} & =\frac{\left[1-(1-\delta)\left(1-\lambda_{2}\right)\right] u_{1}\left(\text { stkat }^{*}\right)}{\left[1-(1-\delta)\left(1-\lambda_{1}\right)\right] u_{2}\left(\text { stkat }^{*}\right)}
\end{aligned}
$$

where

$$
p_{j}^{\prime}(t)=p_{1}(t) b_{1 j}^{-1}+p_{2}(t) b_{2 j}^{-1} \quad j=1,2 \quad \forall t
$$

The second condition is only valid when the utility function is concave at the stationary value and the demands for both goods are strictly positive (interior solutions). If this is not the case, the consumption pattern would be inertial and the consumer would only purchase one good.

Definitions 2.2: A variety-seeking steady-state consumption path for the optimization problem $(\mathrm{P})$ is a steady-state equilibrium with strictly positive consumption of both goods, $q_{1}$ and $q_{2}$. An inertial steady-state consumption path is a steady-state equilibrium in which only one of both goods is consumed.

Proposition 2.1: Consider the dynamic optimization problem $(P)$. When the budget $m(t)$ and the vector price $p(t)$ remain constant over time, the following three possible situations can be presented:

(a).- If $\frac{p_{1}^{\prime}}{p_{2}^{\prime}} \leq \frac{\left[1-(1-\delta)\left(1-\lambda_{2}\right)\right] u_{1}\left(\frac{m b_{11}}{p_{1} \lambda_{1}}\right)}{\left[1-(1-\delta)\left(1-\lambda_{1}\right)\right] u_{2}\left(\frac{m b_{21}}{p_{1} \lambda_{2}}\right)}$, the consumption pattern will converge to an inertial long-run path where only good $q_{1}$ is purchased.

(b).- If $\frac{p_{1}^{\prime}}{p_{2}^{\prime}} \geq \frac{\left[1-(1-\delta)\left(1-\lambda_{2}\right)\right] u_{1}\left(\frac{m b_{1}}{p_{2} \lambda_{1}}\right)}{\left[1-(1-\delta)\left(1-\lambda_{1}\right)\right] u_{2}\left(\frac{m b_{22} 2}{p_{2} \lambda_{2}}\right)}$, the consumption pattern will converge to an inertial long-run path where only good $q_{2}$ is purchased.

(c).- In all other cases the consumption pattern will converge to a variety-seeking long-run path where both goods $q_{1}$ and $q_{2}$ are simultaneously purchased in a fixed proportion.

The expression to the right side of condition $(a)$ is the marginal rate of substitution MRS 
evaluated in the lower edge of the long-run frontier. If this value is higher than the price ratio, the consumer would be better off in the long run by purchasing only good $q_{1}$. The same intuition is valid for condition $(b)$ in the upper edge of the long-run frontier. This edge corresponds to the long-run level of attributes if only $q_{2}$ is purchased. The quasiconcavity assumption imply convex indifference curves, so in all other cases the consumer will be better off by purchasing both goods in every period, and the consumption pattern will converge to a variety-seeking steady state.

The examples from figures $2 \mathrm{a}$ and $2 \mathrm{~b}$ illustrate a variety-seeking and an inertial long-run equilibrium respectively. In the example from figure 1a the interiority condition (c) from proposition 2.1 holds and the stationary stock levels of attributes approached in the long run correspond to an interior equilibrium where the consumer shares the budget between goods 1 and 2 in every period. However, in the example from figure $1 \mathrm{~b}$ both goods are close substitutes in the attribute space, and the non-interior condition (a) from proposition 2.1 holds. When this is the case the agent maximizes his utility by consuming only the good 1 . This inertial consumption plan will eventually converge to the stationary stock level of attributes corresponding to the right side of the long-run frontier.
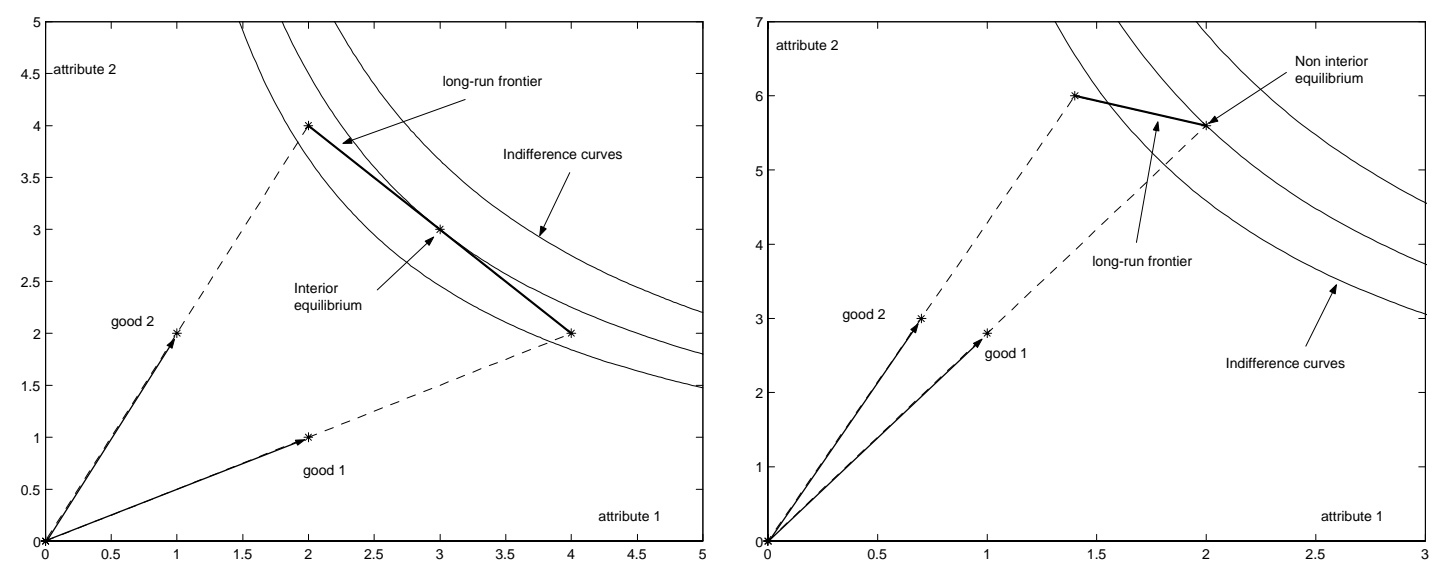

Figures 2a and 2b: Optimal consumption long-run patterns for a logarithmic utility function, $u($ stkat $)=\log ($ stkat $)$. The parameter values are: $m=1, p_{1}=p_{2}=1, \lambda_{1}=\lambda_{2}=0.5$, and $\delta=0.2$. The consumption technology matrixes are $B=\left|\begin{array}{ll}4 & 2 \\ 2 & 4\end{array}\right|$ and $\left|\begin{array}{ll}1 & 0.7 \\ 2.8 & 3\end{array}\right|$ respectively.

\section{The transitional consumption}

The objective in this section is to determine the optimal consumption patterns under several preference structures out of the steady state (e.g. when the consumer tries a new category or a new attribute is available in an established category). For unfamiliar goods the stock levels for 
one or some attributes are much lower that the long-run ones, so some simulations must be run out of the steady state to determine the optimal way in which the consumer accumulates the new attributes.

In an additively separable specification it is assumed that there are no interactions among the attributes. This assumption is implicit in economic models with Cobb-Douglas and logarithmic preferences. In empirical choice models, the additively separable functional form usually produces good predictions and explains a large part of the total variance in empirical research (Dawes and Corrigan 1974; Green and Srinivasan 1978). An additively separable structure can be extended by including additional terms to test for interaction among attributes. From now on, we assume additive separability among the partial contributions to utility made by each constituting attribute. ${ }^{4}$ The utility in period $t$ can be expressed as follows:

$$
u(t)=\sum_{j=1}^{J} u_{j}\left[\operatorname{stkat}_{j}(t)\right]
$$

The utility function assumptions play a central role on the way the consumer behaves when non-familiar attributes are considered in the optimal consumption pattern. On one side, convex utility functions present increasing marginal utilities as consumers accumulate levels of attributes. This convexity reinforces its future consumption, leading to an inertial behavior. On the other side, a concave partial utility presents decreasing marginal utilities. As a result, the utility gain from consuming the same good is every time lower, leading to a variety-seeking behavior. For both preference structures, the marginal utilities increase or decrease monotonically, leading to behavioral patterns that do not depend on the cumulative levels of the level of familiarity with the products. However, for a more complex preference structure, as the inverted-U one proposed by Bawa (1990), an increasing marginal utility region is followed by a decreasing marginal utility, leading to a hybrid behavioral pattern. Under this behavior, consumers who behave inertially for new or unfamiliar items may switch to a variety-seeking behavior for a high level of familiarity. These dynamic processes occur out of a stationary pattern, so in this section some simulations are run to determine the transitional consumption patterns departing from zero levels of attributes for several preference structures.

For the simulations run in this section we assume a cubic partial utility function which

\footnotetext{
${ }^{4}$ According to Johnson, Meyer and Ghose (1989) interactions among attributes are not always statistically significative. They show that adding interaction terms may have a positive effect in the Pearson's validation $r$ when the attributes are highly correlated in the choice set, but in orthogonal settings decreases validation correlations and appears simply to result in "overmodeling."
} 
allows for all the possible behavioral patterns: pure inertia, pure variety seeking and hybrid, as particular cases of the general structure. For expositional purposes we limit the degrees of freedom, setting the following restrictions: the utility function crosses the origin $(u(0)=0)$, the utility range is normalized to the $(0,1)$ interval, and each attribute presents a saturation point at level stkat $=1$. Imposing these conditions, a cubic formulation for the utility function presents one degree of freedom, depending on the marginal utility at stkat $=0$. The resulting formulation is:

$$
u(s t k a t)=(c-2) s t k a t^{3}+(3-2 c) s t k a t^{2}+c s t k a t
$$

where the parameter $c$ is the marginal utility $u^{\prime}($ stkat) at stkat $=0$. When $c=0$ the utility function is convex for stkat $<\frac{1}{2}$. Higher values of $c$ imply a smaller increasing-marginal-utility region. For $c \geq 1.5$ the marginal utility is downward slopping for all the domain stkat $>0$. The implied partial and marginal utilities, and the resulting indifference curves in a two-attribute space are depicted in figures 3 and $4 \mathrm{a}-4 \mathrm{~d}$ respectively for several values of the parameter $c$.
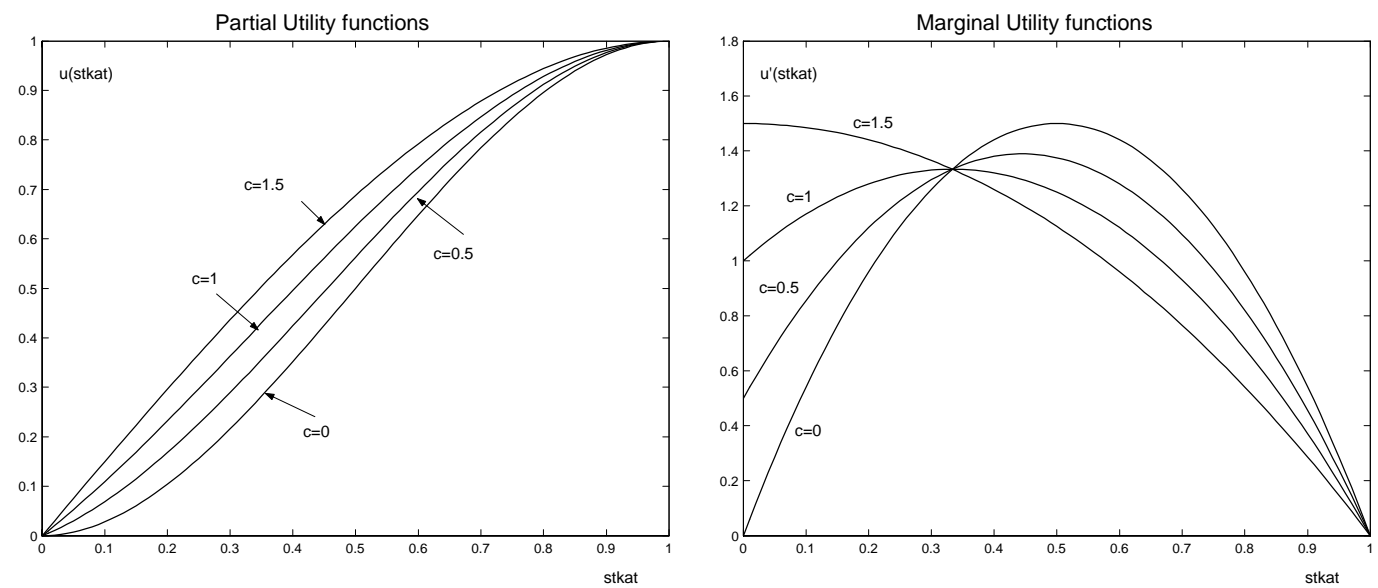

Figure 3: Partial and marginal utility functions for several values of $c$. 


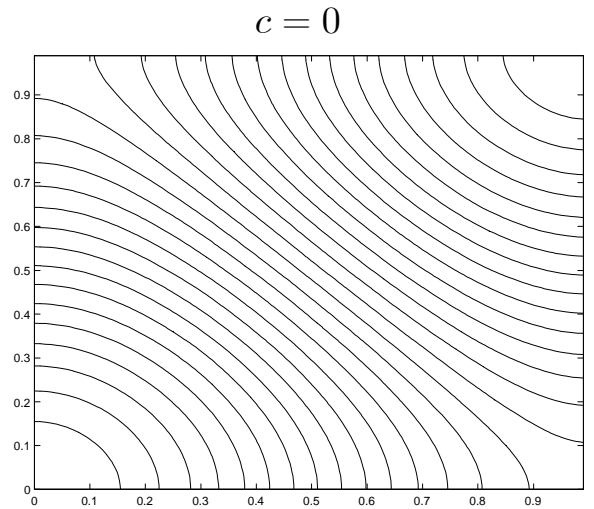

$c=1$

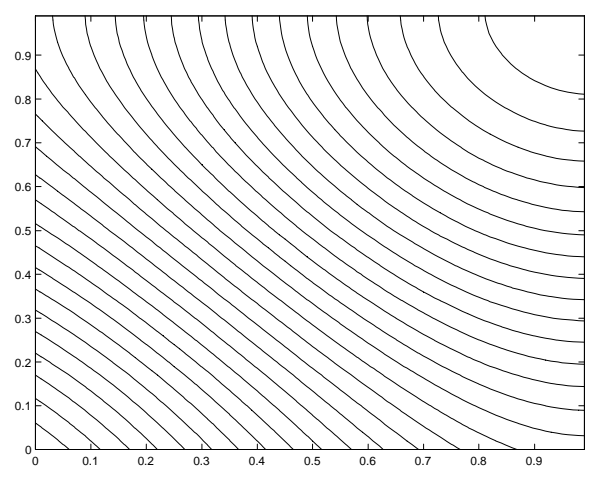

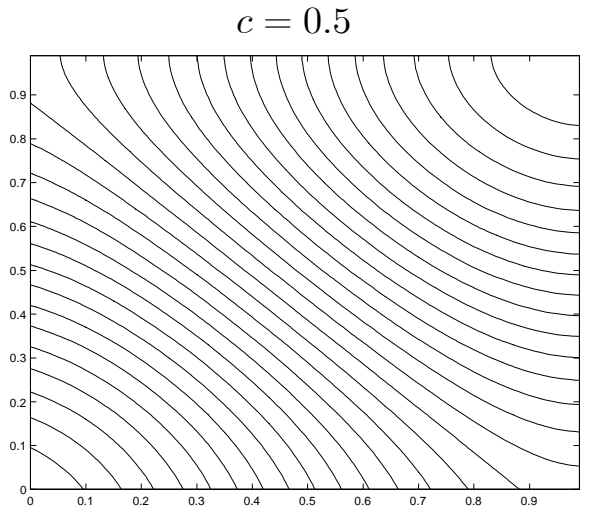

$c=1.5$

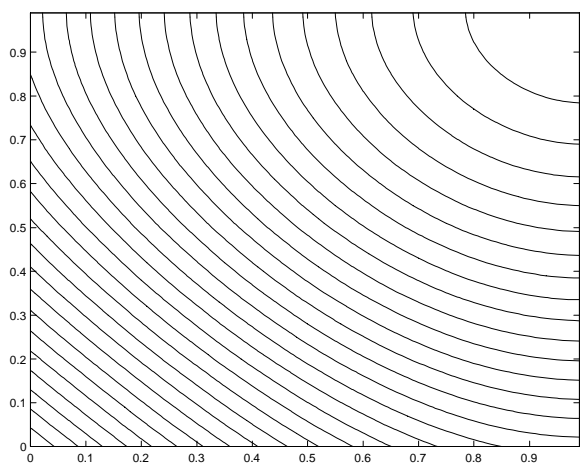

Figures 4a-4d: Indifference curves in a two-attribute space for several values of $c$.

We now run some simulations to determine the optimal consumption path since the consumer tries a new product category. For expositional convenience and without loss of generality, the two products considered in the previous analysis are not multiattribute, so the technology matrix considered in the examples is diagonal. Lets suppose the consumer chooses between two soft drinks: the first one containing coffee, and the second one containing orange juice. The two compounding attributes are caffeine and vitamins. The first drink contains one unit of caffeine, but no vitamins, while the second drink contains one unit of vitamin but no caffeine. In this example the technology matrix considered is diagonal. However, we could alternatively run simulations for a market with two products, each one containing different proportions of both attributes.

The law of motion for the state variables $\left\{\operatorname{stkat}_{1}(t), \operatorname{stk}_{2}(t)\right\}$ is derived by following a standard numerical technique: departing from the steady-state neighborhood, we move backward through the first-order-condition dynamic system (6)-(7). Noteworthy, for some periods far away from the steady state the positivity restrictions in the demands for both goods may be binding even for a quasiconcave region, implying a zero purchase level for one product. If this is the case, condition (7) does not hold and the corner solution is determined by the budget constraint $(6)$. 
The shape of the partial utility function $u($ stkat) conditions the variety-seeking or inertial pattern along the transition, depending on the convexity-concavity of the indifference curves. In all the following examples the long-run equilibrium is a variety-seeking one, as the interiority condition $(c)$ from proposition 2.1 holds, so eventually the consumer will end up buying simultaneously both goods. However, the path departs from a zero level of attribute inventories, so the transitional dynamics illustrates the optimal consumption pattern while the consumer gets used to both attributes.

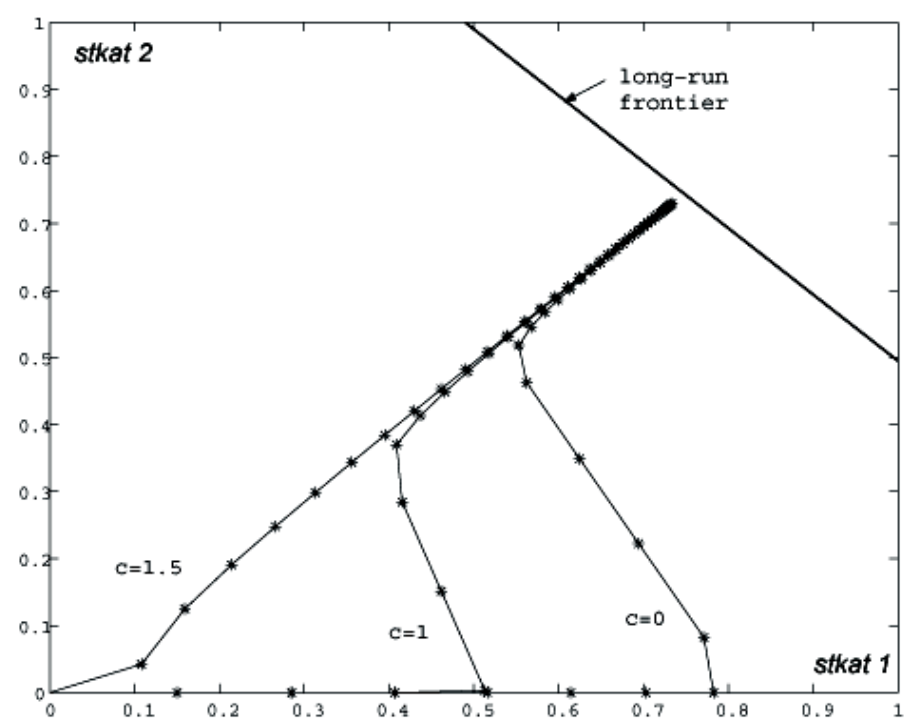

Figure 5: Optimal consumption paths in the attribute space, departing from a zero level of attributes, and the following parameter values: $m=0.15, p_{1}=0.99, p_{2}=1, \lambda_{1}=\lambda_{2}=0.1$, $\delta=0.1, B=\left|\begin{array}{ll}1 & 0 \\ 0 & 1\end{array}\right|$. 

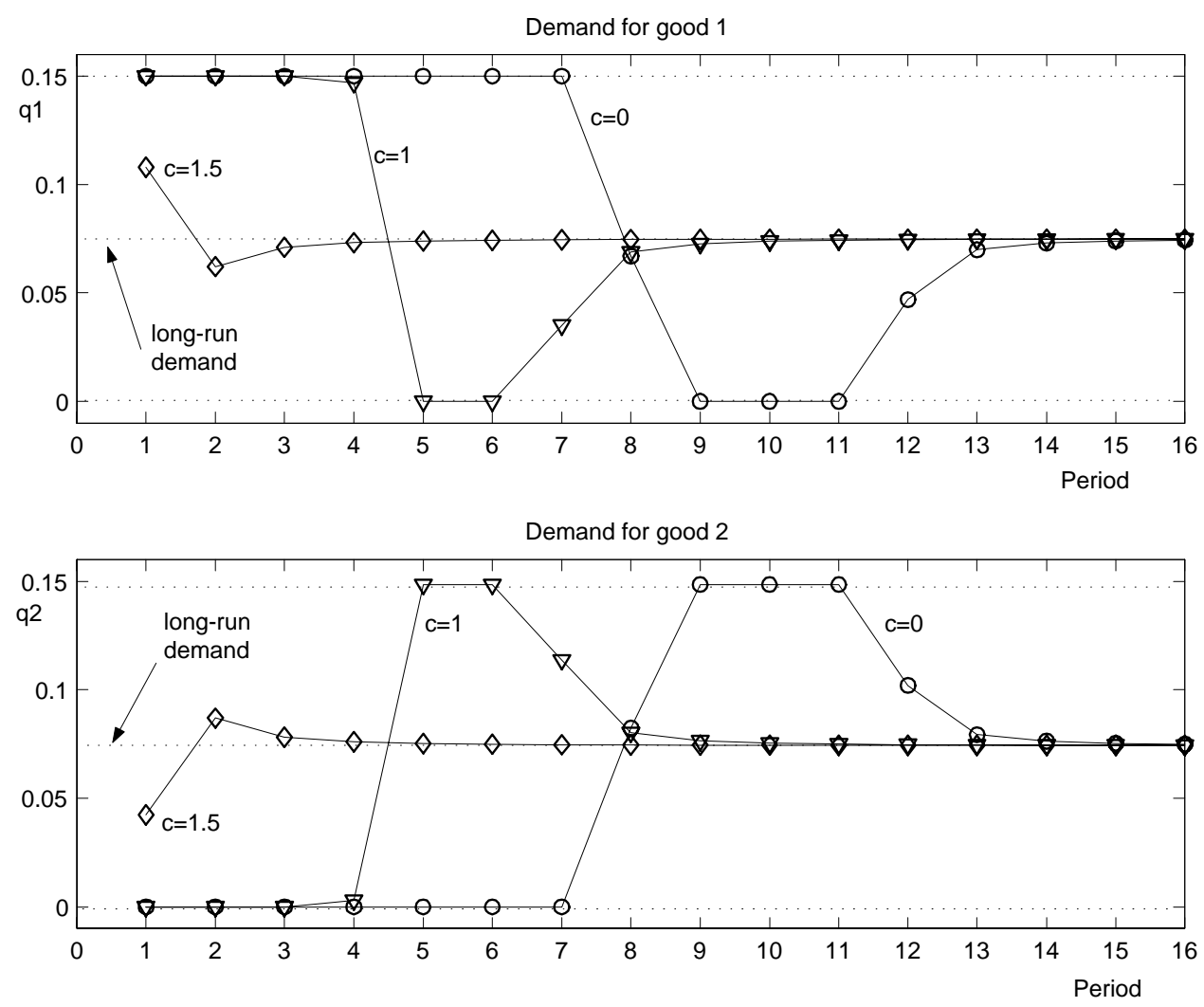

Figure 6: Demands for goods, departing from a zero level of attributes, and the following parameter values: $m=0.15, p_{1}=0.99, p_{2}=1, \lambda_{1}=\lambda_{2}=0.1, \delta=0.1, B=\left|\begin{array}{ll}1 & 0 \\ 0 & 1\end{array}\right|$.

The optimal consumption paths in the attribute space and the implied demands are depicted in figures 5 and 6 respectively for several utility functions. When $c=1.5$ the marginal utility function is decreasing in all the domain $[0,1]$, so every additional unit consumed reduces the next-period marginal utility, discouraging its consumption. Along the whole transition path the consumer splits the budget between both goods and balances both attributes, so the proportion between them gradually approaches the long-run value. As a result, the consumer seeks variety not only in the long run, but also during the first consumptions.

When the utility function is hybrid $(c<1.5)$ the pattern is completely different, as the agent maximizes the utility by consuming only one product and cumulating only one attribute for several periods. Its accumulation fosters its further acquisition as the marginal utility is in the growing region and the indifference curves are concave (see figures 3 and 4). As a result, the consumer behaves inertially by consuming only one good. Switching to the other product will not occur until the discounted marginal utility of acquiring an additional unit is lower than the marginal utility of consuming the first unit of the unknown product. From then on, the consumer will behave inertially with the second attribute, and so on, until the long-run variety-seeking 
equilibrium is approached. As said before, the technology matrix used in the simulations is diagonal, so the products in the analysis are not multiattribute. However, when multiattribute products are considered, the consumer is more likely to choose inertial transitional patterns, as products become substitutes in the attribute space. The closer substitute the products are, the more inertial the consumption plan is.

\section{Indivisible goods and categorical attributes:}

Divisibility of goods and continuous attributes are standard assumptions in most microeconomic models, as the implied demand functions can be best understood if all goods and services are assumed to be fully divisible in the attribute space. ${ }^{5}$ However, the empirical models on consumer choice assume that agents choose among a discrete set of alternative goods compounded of categorical attributes. When consumers decide on the optimal purchase, only few goods like gasoline or electricity can be bought in any quantity desired. For the rest of the goods the consumer is restricted to purchase among a limited set of available products and presentations, so in this section we formulate and analyze the discrete-choice version of the model. We further assume categorical attributes, as most of the compounding attributes of consumers' goods present a categorical nature (e.g. flavor, size, brand, etc.). Indivisibility of goods and categorical attributes are standard assumptions in empirical research on consumer choice. These statements bring reality to both the purchase and consumption processes and are of special interest for understanding the individual demand for most frequent-consumption categories.

In the discrete version of the problem, every period the agent is restricted to choose among a limited set of alternatives to be consumed. Consistent with the choice models with indivisible goods, at every period $t$ the agent derives utility from consuming any of the $I$ available indivisible goods, $x_{1}, x_{2}, \ldots, x_{I} \in X$, for a given category. Let's further assume that the consumer can afford any of the consumption alternatives, $p_{x_{i}}(t) \leq m(t) \forall t, \forall x_{i} \in X$.

Each categorical attribute $j$ (e.g. flavor), is characterized by a set of $K_{j}$ categories (e.g. $1=$ strawberry, $2=$ lemon, $3=$ vanilla, $\ldots, K_{j}=$ chocolate). If we assumed that consumer preferences for a product like a yogurt depend exclusively on flavor, it would be required a $K_{j}$ dimensional space (one dimension for each flavor) to define the state of a consumer, based on previous

\footnotetext{
${ }^{5}$ See Lancaster (1971) for a detailed exposition and analysis of the attribute-based microeconomic models.
} 
consumption. Therefore, for each attribute $j$ we define the state-dependence variable, $s t k a t_{j}$, which is a vector of length equal to the $K_{j}$ categories, that accounts for the cumulative stock of every category of attribute. The element $s t k a t_{j k}$ is the cumulative stock level of category $k$ for attribute $j$. The corresponding consumption technology for good $x_{i}$ is captured by a binary vector $b_{j i}$ of length equal to the $K_{j}$ categories of attribute $j$. The element of this vector $b_{j i k}$ corresponding to the category $k$ included in the item $i$ is equal to 1 and the remaining elements equal 0 . In our example, the vector $b_{j i}$ corresponding to the attribute $j$ (flavor) for a lemon yogurt $(k=2)$ would be $(0,1,0,0, \ldots 0)$.

As in the previous sections, the utility function is additively separable in the attribute space. It is also assumed that utility from consuming a good is determined by the consumer state determined by the cumulative levels of each of the $K_{j}$ categories of each attribute $j$. In every period $t$ the agent derives utility from the stock level of each category of attribute $s t k a t_{j k}$ :

$$
u_{x_{i}}(t)=\sum_{j=1}^{J} \sum_{k=1}^{K_{j}} u_{j k}\left[\operatorname{stkat}_{j k}(t)\right]
$$

where the stock of category $k$ of attribute $j$ derived from consumption of good $x_{i}$ is determined by the law of motion:

$$
\operatorname{stkat}_{j k}(t)=b_{j i k}+\left(1-\lambda_{j}\right) s t k a t_{j k}(t-1) \quad \forall i, \forall j, \forall k, \forall t
$$

By plugging equation (11) into the summation, the partial utility derived from consumption of good $x_{i}$ at time $t$ can be expressed as a function of the depreciated level of attribute categories $\left(1-\lambda_{j}\right) s_{k} t_{j}(t-1)$ plus the additional units of attributes, provided by product $x_{i}$ :

$$
u_{x_{i}}(t)=\sum_{j=1}^{J} \sum_{k=1}^{K_{j}} u_{j k}\left[\left(1-\lambda_{j}\right) \operatorname{stkat}_{j k}(t-1)+b_{j i k}\right]
$$

The partial utility functions can be approximated by a first-order Taylor expansion from the depreciated levels of attribute categories from previous period, $\left(1-\lambda_{j}\right) s t k a t_{j k}(t-1)$ :

$$
u_{x_{i}}(t) \simeq \sum_{j=1}^{J} \sum_{k=1}^{K_{j}} u_{j k}\left[\left(1-\lambda_{j}\right) s t k a t_{j k}(t-1)\right]+\sum_{j=1}^{J} b_{j i} u_{j k}^{\prime}\left[\operatorname{stkat}_{j}(t)\right]
$$

The first summation in the previous equation is common for every alternative good and represents the baseline utility derived from the depreciated levels of attribute categories. The 
second summation represents the additional utility derived from the attributes contained in good $x_{i}$. The utility gain leads to the same preference structure and implied indifference curves, so without loss of generality the utility when consuming product $x_{i}$ at period $t$ can be expressed as the summation of the marginal utilities derived from the additional amounts of the compounding attribute categories. In line with the previous section, for the partial utility functions $u($ stkat), we assume a general cubic formulation that nests the inertial, variety seeking, and hybrid behaviors as particular cases. The second summation from the previous equations remains as follows:

$$
u_{x_{i}}(t) \simeq \sum_{j=1}^{J} b_{j i} u_{j}^{\prime}\left[\operatorname{stkat}_{j}(t)\right]=\sum_{j=1}^{J} b_{j i}\left[\alpha_{o j}+\alpha_{1 j} \operatorname{stkat}_{j}(t)+\alpha_{2 j} s t k a t_{j}^{2}(t)\right]
$$

As in the previous section, some simulations were run to determine the consumption pattern, departing from a zero level of attributes. Formally, given the initial stock of attributes, stkat ${ }_{j 0}$, the consumer will choose the optimal consumption sequence for the $t$ periods to maximize the utility at every period, subject to the stocking processes for every attribute and the consumption technology:

$$
\max _{x_{i}(t) \in X} \sum_{t=1}^{\infty}(1-\delta)^{t} \sum_{j=1}^{J} b_{j i}\left[\alpha_{o j}+\alpha_{1 j} s t k a t_{j}(t)+\alpha_{2 j} s t k a t_{j}^{2}(t)\right] \quad t=1,2, \ldots
$$

subject to:

$$
\begin{gathered}
\operatorname{stkat}_{j}(t)=b_{j i}+\left(1-\lambda_{j}\right) \operatorname{stkat}_{j}(t-1) \quad \forall i, \forall j, \forall t \\
\operatorname{stkat}_{j}(0)=\operatorname{stkat}_{j 0} \quad \forall j
\end{gathered}
$$

In this version of the model with indivisible goods, a consumption pattern for a limited horizon of $t$ periods is a sequence $\{x(1), x(2), x(T)\}, \quad x(t) \in X$ where the agent selects one of the possible $I$ products in each period. When the choice set is discrete, the dynamic programming techniques using first-order conditions can not be used to characterize the optimal solution, and the demand functions have to be determined by comparing the derived utility from choosing any of the $I^{T}$ possible patterns. As in previous section, for expositional purposes we restrict the marginal utility function (12) to the functional form from equation (10). For every period the consumer is assumed to choose among two existing products, fully characterized by an attribute (e.g. flavor) with two categories (e.g. strawberry and vanilla), each one contained in one product. A time horizon of 20 periods and a inverted-U marginal utility function $(c=0)$ are also assumed. For every period, the budget and the price for each product are set to one $\left(m=p_{1}=p_{2}=1\right)$. 
We have analyzed two different cases corresponding to different consumption technologies. The amount of attribute in case $a$ is lower than in case $b$, so it will take more periods for a consumer in case $a$ to accumulate the same level of attributes than in case $b$. The rest of the parameters remain equal in both cases. In order to analyze the effect of rational agents considering future utilities, we have run simulations for every possible value of the future discount parameter $\delta$. The demands for both goods and the implied optimal patterns in the attribute space are depicted in figure 7 .

$$
\text { Case } a: \quad B=\left|\begin{array}{cc}
0.23 & 0 \\
0 & 0.23
\end{array}\right|
$$
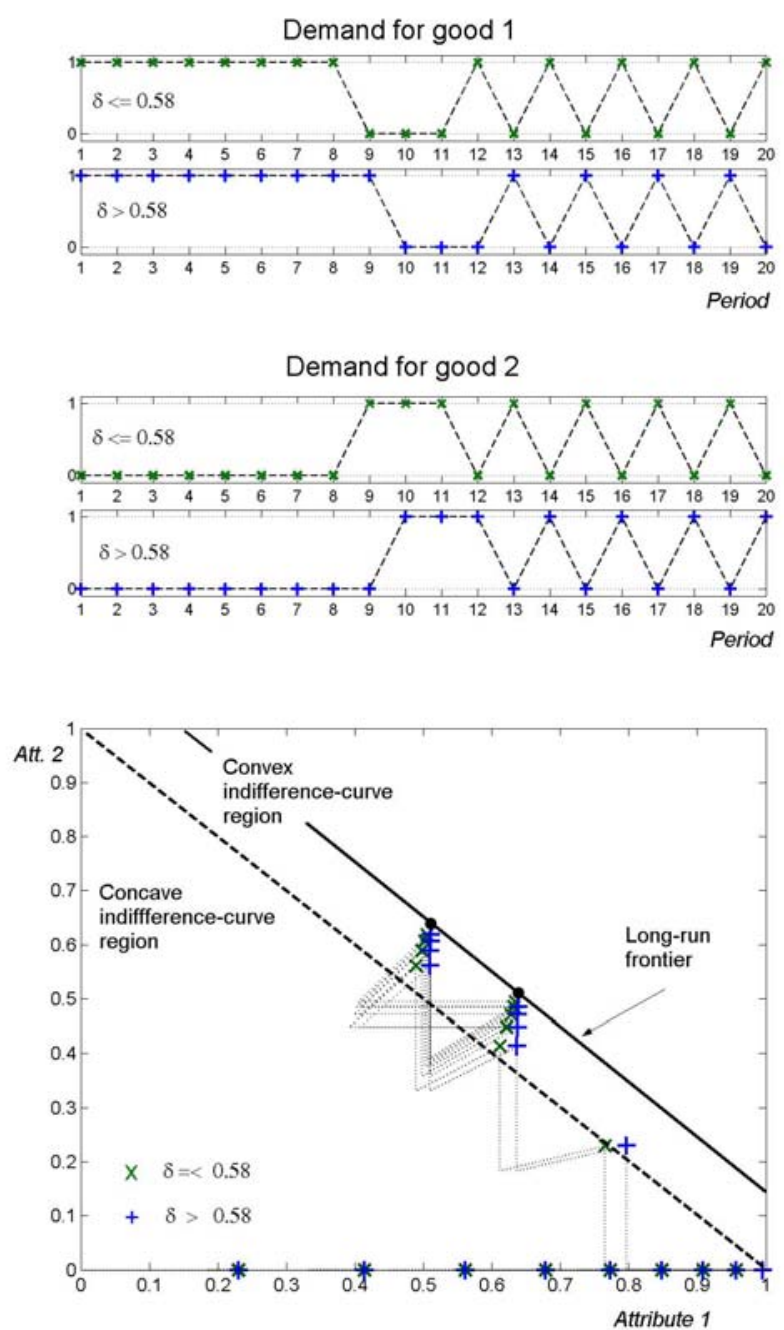

Case $b: \quad B=\left|\begin{array}{cc}0.33 & 0 \\ 0 & 0.33\end{array}\right|$
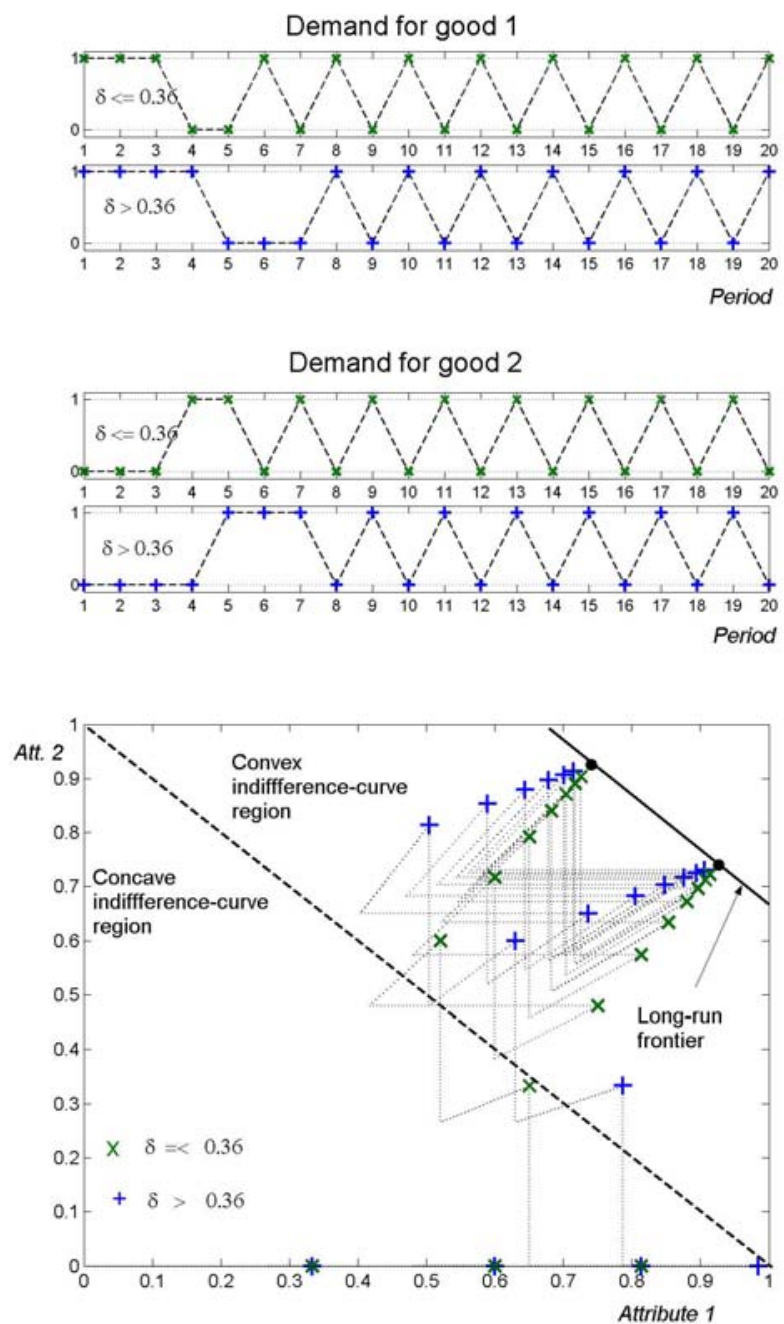

Figure 7: Demands for goods and optimal path in the attribute space, departing from a zero level of attributes, and the following parameter values: $m=1, p_{1}=p_{2}=1, \lambda_{1}=\lambda_{2}=0.2$, $c=0, T=20$.

In the previous section the goods were assumed to be divisible and the agent seeks variety by consuming simultaneously both products. However, simultaneous consumption of several goods 
is not possible in the indivisible-good version, and the resulting variety-seeking behavior implies switching among the existing items. The optimal paths present a similar qualitative behavior than the ones obtained for the continuous-choice-set version studied in the previous sections. As expected, when a inverted-U marginal utility function is considered for both categories of attributes the optimal consumption path follows the same hybrid pattern that the one presented in the divisible-good framework. The consumer behaves inertially during several periods until a high level of familiarity with the product containing the implied category is reached. At that level the decreasing marginal utility regions have been reached and the agent switches to consume a new unfamiliar product during several periods. Once the level of familiarity with the available products is high, the consumer shifts among them from period to period, seeking variety. As the finite horizon $T$ increases, the consumption pattern converges to a long-run equilibrium where the consumer alternates purchases between the two products.

Noteworthy, the future-discount parameter $\delta$ does not affect the optimal consumption pattern in a significant way. The results are qualitative similar for every possible value in the domain $(0$, 1], although forward-looking agents switch to the variety-seeking pattern earlier. A myopic agent $(\delta=1)$ will behave inertial, consuming good 1 until reaching the convex-indifference region. The optimal path is the same for consumers with a future-discount parameter $\delta>0.58$ and $\delta>0.36$ in cases $a$ and $b$ respectively. However, forward-looking agents with low future discount rates $(\delta<0.58$ and $\delta<0.36$ in cases $a$ and $b$ respectively) switch to product 2 one period earlier. As a result, the variety-seeking behavior is approached earlier. This pattern allows forwardlooking agents to invest in the second attribute earlier, sacrificing present utility in the concave indifference-curve region for a higher future utility when being in the convex indifference-curve one.

\section{Empirical Illustration}

The objective of this section is to empirically illustrate the attribute-based consumer choice model studied in the previous section for a myopic agent. We use three different panels of households. The first scanner dataset, previously used by Fader and Hardie (1996; hereafter F\&H), contains information on 9781 purchases of fabric softener over a $2 \frac{1}{2}$-year period (January 1990 to June 1992) among 594 households. The second dataset, previously used by Jain, Vilcassim, and Chintagunta (1994), and Roy, Chintagunta, and Haldar (1996), consists of 300 households 
making 2,798 purchases of catsup. The third dataset, also used by the former authors, contains information on 2,509 purchases of saltine crackers among 100 households.

Additional information regarding prices and retail promotional activities for every item is also available at every purchase occasion. All the datasets include the following set of marketing variables for each item: price, special display, and newspaper feature advertisement. The four compounding categorical attributes in the fabric softener market are brand (Arm \& Hammer, Bounce, Cling Free, Downy, Final Touch, Generic, Private Label, Snuggle, Sta Puff, and Toss n'Soft), form (concentrated, refill, liquid, and sheets), formula (light, regular, staingard, and unscented) and size (small, medium, large, and extra-large). The two compounding attributes in the catsup market are brand (Heinz and Huntz) and size (28, 32, and 40). The 16-ounce saltine cracker category consists of three major national brands (Sunshine, Keebler, and Nabisco) and the remaining local brands, grouped together under "private labels" in the analysis.

The standard approach to model product choice with scanner data considers the multinomial logit (MNL) model (see McFadden 1974, Guadagni and Little 1983, Lattin 1987, and F\&H among others). The MNL model computes the probability of choosing an alternative as a function of the utilities of all the available alternatives. Given some standard assumptions it can be shown that the MNL model has the following functional form:

$$
p_{i}=\frac{e^{\nu_{i}}}{\sum_{J} e^{\nu_{j}}}
$$

where, ignoring household $h$ and time $t$ indices, $p_{i}$ is the probability of choosing item $i$, and $\nu_{i}$ is the deterministic component of item $i$ 's utility. ${ }^{6}$

We consider two sources of heterogeneity: as Bucklin and Gupta (1992), Chintagunta (1992) and Fader and Hardie (1996) we consider heterogeneity between segments of consumers; and, as these and many other authors we consider heterogeneity within a segment of consumers. The heterogeneity among consumers in a given segment is captured by the state-dependence vector stkat that accounts for the cumulative stock of each category of attribute. We normalize our variable stkat to lie within the $(0,1)$ interval by using the loyalty measure used in Guadagni and Little (1983). ${ }^{7}$

\footnotetext{
${ }^{6}$ Following McFadden (1974), the conditional probability equations can be obtained by using a random utility framework and assuming that the stochastic components of utility are independent and identically distributed for each alternative as double exponential. This random utility assumption has been widely used in choice modelling.

${ }^{7}$ Guadagni \& Little (1983) use the following exponentially smoothing formulation to weight the past purchase history on brand and size attributes for each household:

$L O Y_{h j}(t)=\lambda i_{h j t}+(1-\lambda) L O Y_{h j}(t-1)$

It is worth noting, by comparing the previous equation to equation (11), that the loyalty variable $L O Y$ is a
} 
Our premise holds that the utility of an item is given by the sum of the partial utilities provided by the constituent attribute levels. Assuming no interactions, item $i$ 's utility, $\nu_{i}$, can be expressed as additively separable partial functions of the stock levels of the $J$ constituent attributes from equation (12) and the values of the marketing variables for item $i, Z_{i}{ }^{8}$ The utility function for the item $i$ in the consumer's segment $s$ is:

$$
\nu_{i}^{S}(t)=\sum_{j=1}^{J} \beta^{S} Z_{i}(t)+b_{j i}\left[\alpha_{o j}^{S}+\alpha_{1 j}^{S} s t k a t_{j}(t)+\alpha_{2 j}^{S} s t k a t_{j}^{2}(t)\right] \quad \forall t, \forall x_{i} \in X
$$

where the remaining $\beta Z_{i}$ term includes the standard set of marketing variables: price and two dummy variables indicating promotional activities: displays and newspaper features. The vector $b_{j i}$ is the binary one of length equal to the $K_{j}$ categories of attribute $j$. As in the previous section, the element of this vector corresponding to the category included in the item $i$ is equal to 1 , and the remaining elements equal 0 . The vector $\alpha_{0 j}$ includes the intercept terms for the $K_{j}$ categories of attribute $j$ and the parameters $\alpha_{1 j}$ and $\alpha_{2 j}$ correspond to the quadratic functional form proposed for the stock level of the attribute. This formulation is a natural extension of the linear specification used by F\&H.. However, our quadratic specification allows for several consumption patterns for each attribute: pure inertia, pure variety seeking, and hybrid behavior.

Up to this point, both the choice occasion and household indices have been suppressed. Let them be defined as $t$ and $h$ respectively, and let $H$ be the number of households in the panel, $T_{h}$ the number of choice occasions for household $h$, and $\delta_{i t}^{h}$ be a purchase indicator equal to 1 if household $h$ chooses the item $i$ on purchase occasion $t$, and 0 otherwise. For the model specification with $S$ preference segments or latent classes, the model parameters are estimated by maximizing the following Log-Likelihood function:

$$
L L=\sum_{h=1}^{H} \ln \left[\sum_{s=1}^{S}\left(\prod_{t=1}^{T_{h}} \prod_{i} p_{i / s}^{h}(t)^{\delta_{i t}^{h}}\right)\right]
$$

As in Fader and Hardie (1996), the number of segments is selected by carrying out the estimation of an increasing number of segments until there is no significant improvement of model adjustment. As in the previous literature on MNL mixture model (Kamakura and Russell 1989, Bucklin and Gupta 1992, and Fader and Hardie 1996) we choose between different models

normalized measure of the stock level of the implied attribute, stkat. When the investment from the previous equation is normalized to 1 , the maximum stock level attainable after infinite periods of consecutive investment in the attribute is 1 , and the previous-period stock of attribute depreciates at a rate $\lambda$.

${ }^{8} \mathrm{As}$ in the case with most linear models, this additively separable specification can usually produce good predictions and explains a large part of the total variance (Dawes and Corrigan 1974; Green and Srinivasan (1978); Johnson, Meyer and Ghose 1989). However, interactions can easily be included in the model, but because of the large number of potential interaction effects, the process of adding interaction terms should be driven according to the knowledge of the product category. 
on the basis of the Akaike Information Criterion (AIC). ${ }^{9}$ We also report a likelihood ratio test for nested models.

\subsection{Analysis of the results}

Table 1 reports the results from our calibration exercise. [The complete set of results is available on request]. For every market we report two sets of results: the one using the non-linear specification from equation (13), and the nested linear state-dependence structure $\left(\alpha_{2}=0\right)$, proposed by Fader and Hardie (1996). Additionally, for the multiple-segment models we consider two nested specifications: one with restricted-across-segments depreciation parameters $\lambda_{j}$ (specification $[\mathrm{R}]$ ), and another with free depreciation parameters (specification $[\mathrm{U}]$ ).

Comparing multiple-segment specifications, in all the three markets the three-segment model provides the best fit of the data since it has significantly better evaluation criteria. One open question is the specification of the depreciation parameters. The Log-likelihood ratio test and the AIC show that the three-segment unrestricted depreciation parameters model [U] best fits the data for the fabric softener and catsup markets, while the three-segment restricted model $[\mathrm{R}]$ is a preferred specification for the cracker market. We also test for the significance of the nonlinear structure. In all the selected models, the AIC criterion favors the non-linear specification. Except for the case of the catsup market (p-value=0.07) all the likelihood ratio tests strongly reject the linear specifications in favor of the non-linear ones.

\footnotetext{
${ }^{9}$ The AIC is defined as $-2 \log L+2 k$, where $k$ is the number of parameters and $N$ is the number of observations.
} 
Table 1: Calibration results by Product category

Fabric softener

\begin{tabular}{|c|c|c|c|c|c|c|c|c|}
\hline \multirow[b]{3}{*}{$1 \mathrm{seg}}$. & \multicolumn{3}{|c|}{ Linear specification } & \multicolumn{3}{|c|}{ Non-lin. specification } & \multicolumn{2}{|c|}{$\begin{array}{l}\text { Godness of fit } \\
\text { (Lin vs. Non.lin) }\end{array}$} \\
\hline & d.f. & $\mathrm{LL}$ & $\mathrm{AIC}$ & d.f. & $\mathrm{LL}$ & AIC & d.f. & \\
\hline & 30 & -10987.1 & 22034.2 & 34 & -10957.2 & 21982.4 & 4 & $59.781(0.000)$ \\
\hline 2 seg. $[R]$ & 57 & -10798.0 & 21710.1 & 65 & -10636.3 & 21402.2 & 8 & $323.46(0.000)$ \\
\hline 2 seg. [U] & 61 & -10790.1 & 21702.2 & 69 & -10627.3 & 21392.6 & 8 & $619.20(0.000)$ \\
\hline 3 seg. [R] & 84 & -10566.4 & 21300.8 & 96 & -10480.9 & 21153.8 & 12 & $292.41(0.000)$ \\
\hline 3 seg. [U] & 92 & -10550.2 & 21284.4 & 104 & -10459.5 & $21127.2^{*}$ & 12 & $314.45(0.000)$ \\
\hline
\end{tabular}

* Preferred specification

Observations 9781. Number of consumers: 594. First 3227 purchases from year 1991 reserved for att. level initialization.

\section{Catsup}

\begin{tabular}{|c|c|c|c|c|c|c|c|c|}
\hline & \multicolumn{3}{|c|}{ Linear specification } & \multicolumn{3}{|c|}{ Non-lin. specification } & \multicolumn{2}{|c|}{$\begin{array}{c}\text { Goodness of fit } \\
\text { (Linear vs. Non.lin) }\end{array}$} \\
\hline & d.f. & $\mathrm{LL}$ & $\mathrm{AIC}$ & d.f. & $\mathrm{LL}$ & $\mathrm{AIC}$ & d.f. & $\chi^{2}(\mathrm{P}$-value $)$ \\
\hline $1 \mathrm{seg}$. & 10 & -1389.02 & 2798.04 & 12 & -1388.39 & 2800.78 & 2 & $1.26(0.53)$ \\
\hline 2 seg. $[R]$ & 19 & -1349.38 & 2736.76 & 23 & -1345.72 & 2737.44 & 4 & $7.32(0.12)$ \\
\hline 2 seg. [U] & 21 & -1343.60 & 2729.20 & 25 & -1341.73 & 2733.46 & 4 & $3.74(0.44)$ \\
\hline $3 \mathrm{seg} .[\mathrm{R}]$ & 28 & -1331.23 & 2718.46 & 34 & -1324.21 & 2718.60 & 6 & $14.04(0.03)$ \\
\hline 3 seg. [U] & 32 & -1325.30 & 2716.64 & 38 & -1319.70 & $2714.94^{*}$ & 6 & $11.20(0.07)$ \\
\hline
\end{tabular}

\section{Cracker}

\begin{tabular}{|c|c|c|c|c|c|c|c|c|}
\hline \multirow{2}{*}{ 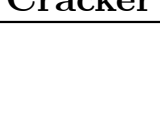 } & \multicolumn{3}{|c|}{ Linear specification } & \multicolumn{3}{|c|}{ Non-lin. specification } & \multicolumn{2}{|c|}{$\begin{array}{l}\text { Godness of fit } \\
\text { (Lin vs. Non.lin) }\end{array}$} \\
\hline & d.f. & LL & $\mathrm{AIC}$ & d.f. & LL & $\mathrm{AIC}$ & d.f. & $\chi^{2}(\mathrm{P}$-value $)$ \\
\hline $1 \mathrm{seg}$. & 8 & -1241.24 & 2498.48 & 9 & -1239.91 & 2497.82 & 1 & $2.66(0.102)$ \\
\hline 2 seg. $[R]$ & 16 & -1207.35 & 2446.70 & 18 & -1207.27 & 2450.54 & 2 & $0.16(0.923)$ \\
\hline 2 seg. [U] & 17 & -1206.52 & 2447.04 & 19 & -1206.51 & 2451.02 & 2 & $0.02(0.990)$ \\
\hline 3 seg. $[R]$ & 24 & -1195.77 & 2439.54 & 27 & -1177.11 & $2408.22^{*}$ & 3 & $37.32(0.000)$ \\
\hline 3 seg. [U] & 26 & -1181.44 & 2414.88 & 29 & -1176.01 & 2410.02 & 3 & $10.86(0.012)$ \\
\hline
\end{tabular}

* Preferred specification

Observations 3292. Number of consumers: 126. First 5 observations from each consumer reserved for attribute level initialization. 
The utility of consuming a good $x_{i}$ depends on three effects captured in equation (13): relating to marketing, attribute intercepts, and attribute stock levels. The effect of the first set of marketing variables: price, displays, and newspaper features, is captured by the vector of parameters $\beta$. The other two sets of explanatory variables are related to the set of product attributes and the past purchase history. The partial contribution to utility for each constituent attribute present in the dataset is decomposed in two effects: the preference for each category $k$ of attribute $j$, captured by the vector of $K_{j}$ intercept terms, $\alpha_{0 j}$; and the cumulative-stock effect for attribute $j$, captured by the quadratic expression, $\alpha_{1 j} s t k a t_{j}+\alpha_{2 j} s t k a t_{j}^{2}$. Both effects vary across segments. The first effect captures the relative preferences for the several categories of attributes. The second captures the additional source of heterogeneity across consumers within a segment as a result of the previous purchase history (state-dependence effect).

Considering multiple segments allows for capturing the significant heterogeneity on preferences across agents. For all the three markets, the segments present significant differences for every set of parameters, capturing the several sources of heterogeneity across consumers. In this analysis we focus on the state-dependence behavior which is the central issue of our model, captured by the partial functions $\alpha_{1 j} s t k a t_{j}+\alpha_{2 j} s t k a t_{j}^{2}$. The partial utilities by segment and attribute are illustrated in figure 8 . 


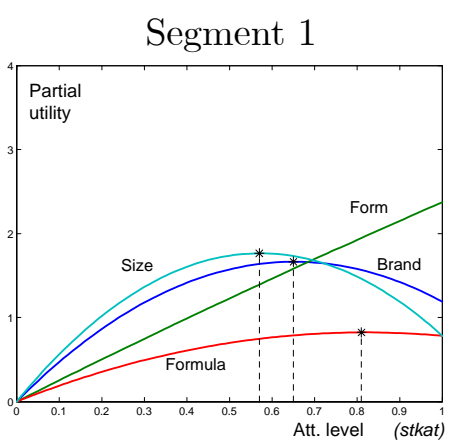

Segment 1

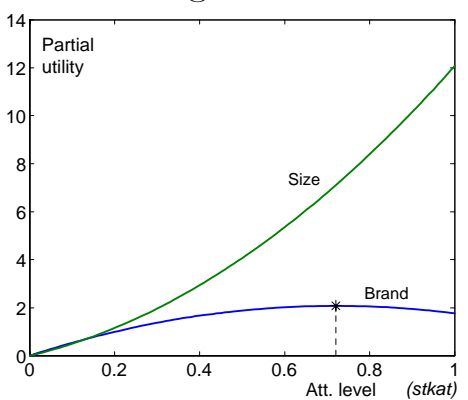

Segment 1

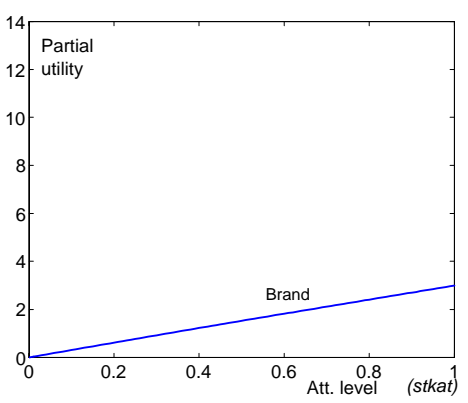

Fabric softener Market Segment 2

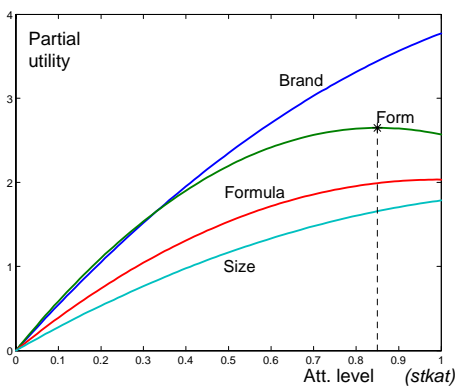

Catsup Market Segment 2

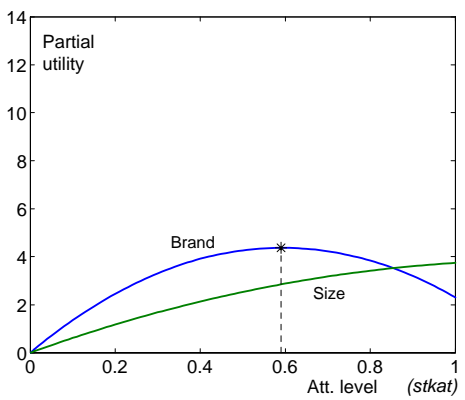

Cracker Market Segment 2

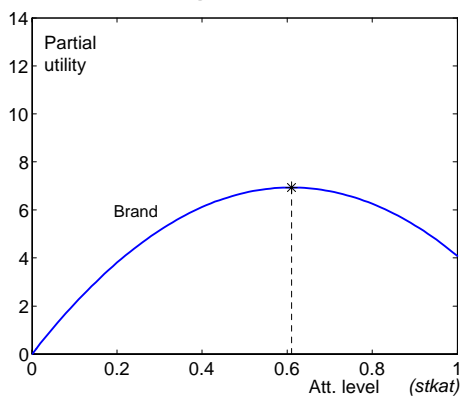

Segment 3

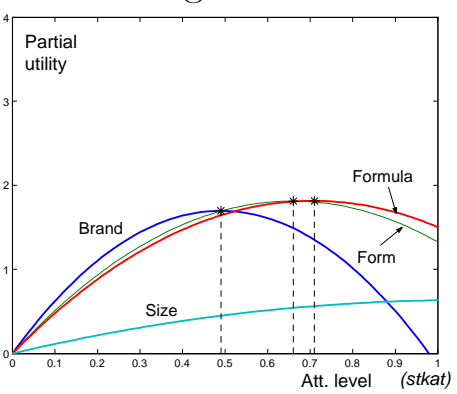

Segment 3

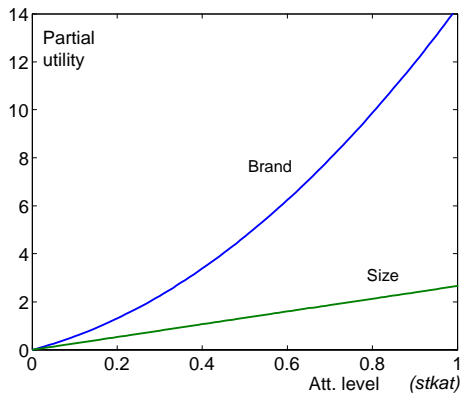

Segment 3

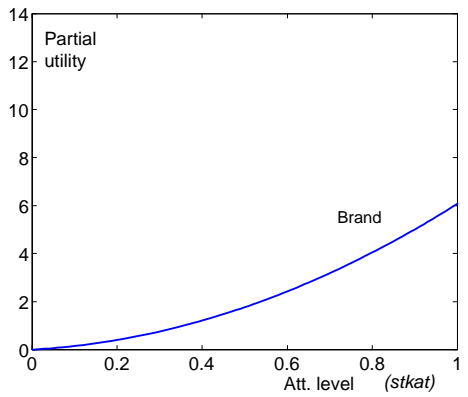

Figure 8: Partial utility functions for each attribute, by segment.

In relation to the state-dependent behavior, we find strong evidence of inertial behavior for low levels of attributes. The linear parameters $\alpha_{1}$ are all positive and significantly different from 0 at a $5 \%$ level for all attributes, all markets, and all segments. This result implies a consistent inertial behavior for low cumulative levels of attributes, as acquiring products with unfamiliar characteristics consistently reinforces its further acquisition.

We also find strong evidence of non-linear behavior for most attributes. A total of 16 out of the 21 estimated parameters $\alpha_{2}$ are negative and significantly different from 0 at a $5 \%$ level for most attributes and market segments. These values imply strictly concave partial utility functions. The concave utility functions present steeper slopes for the partial utilities at a 
zero level of attribute. In line with our theoretical model, this suggests maximum positive slopes and strong inertial purchasing patterns for new or unstocked attributes. Due to the concavity of the utility functions presenting $\alpha_{2}<0$, this reinforcement of previously purchased categories becomes weaker as the cumulative levels increase. However, for a hybrid behavior to be presented, the estimated function must present a maximum and decreasing region on the domain $(0,1)$. As the partial utility reaches a maximum, the critical level is overcome. Exceeding this critical value, every new acquisition of the attribute category reduces the probability of acquiring an item containing it in the new purchase, so the consumer starts to seek variety for this attribute category. The 10 critical levels for a switching behavior, when present, are depicted in dashed lines in figure 8.

Using the depreciation parameters estimated $\lambda_{j}$ for each segment, it is possible to determine the required number of consecutive purchases for an item, containing the same category of attribute $j$, to switch from inertia to variety seeking. To illustrate the switching effect, let us assume that a new young consumer from segment 1 starts in the habit of using fabric softeners, departing from a zero level of attributes. According to the depreciation parameter for the attribute brand and the partial utility function estimated, after the fourth purchase, the brand level variable reaches a value of 0.7509 , surpassing the critical value $s t k a t^{*}=0.6476$ (see the dashed lines in figure 8). The consumer is now less likely to buy the same brand than in the previous purchase, thus seeking variety in this attribute. The same switching behavior occurs after the fourth consecutive purchase of a product of the same size, or after the sixth consecutive purchase of a product containing the same formula. In contrast, for the form attribute, consumers in segment 1 present strong inertia, as past purchases of an item continuously reinforce future purchases of a product with the same form. After becoming familiar with the several fabric softeners, an experienced consumer in segment 1 may switch among the preferred brands, formulas, and sizes, while behaving form loyal.

As illustrated in the partial utilities from figure 8 , in the fabric softener market, segments 1 and 3 present a strong hybrid behavior for most attributes, while segment 2 behaves inertial for all attributes but form. In the catsup market all the three segments present strong inertia for the attribute size, while segments 1 and 2 present hybrid behavior for brand. Segment 2 in the cracker market also presents a hybrid behavior for the only attribute brand. 


\section{Conclusions}

This research presents an attribute-based dynamic model where rational consumers derive utility from the attribute inventories accumulated when items are consumed. In light of the empirical evidence of several state-dependence patterns, the model considers a general specification of the utility function that allows for the different types of behavior described in the literature: pure inertia, pure variety seeking and hybrid. The transitional dynamics and the steady-state conditions are characterized. Depending on the utility assumptions, the model may present a stationary consumption pattern that can be inertial, where the consumer only purchases one product, or a variety-seeking one, where the consumer buys several products simultaneously. The conditions for the inertial or variety-seeking steady state equilibrium are also determined.

The analysis of the transitional dynamics of the model is key to understand new-consumer patterns while getting used to the product category in growing markets as well as experiencedconsumer reactions to a significant change in the market. The response of a regular and experienced consumer to a market change is also driven by the transitional dynamics. For instance, an innovation adopted in a frequently purchased product category, like the launch of a new packaging technology, if properly promoted, may induce trial, with a long-term effect as an inertial consumer will eventually change habits in the long run, converging to a new inertial steady state.

The model proposed in this research considers two inter-temporal effects. The first one is produced by past consumption affecting the present utility through the cumulative stock of attributes. The second effect is originated by assuming forward-looking agents that discount the flow of future utilities when taking present decisions. For a hybrid utility function, forwardlooking agents behave qualitatively similar to the myopic ones, but switch earlier from the inertial behavior to the variety-seeking one. However, depending on the preference structure, forwardlooking assumptions may have relevant implications in the transitional dynamics and is a major issue to be considered in our future research. For instance, state-dependence and forward-looking assumptions are both key for understanding consumption of habit-creation products like alcohol or tobacco.

We run an empirical application with scanner datasets for several convenience goods. For this exercise we estimated the simplified version of the model for the myopic-agent case. The empirical results lend strong support to the importance of modeling consumer preferences in the attribute space in a manner that captures the dynamic accumulation of the compounding 
characteristics. The choices among the existing items reveal preferences not only for a brand, but also for several other underlying attributes, like size, formulas, flavors, etc. Our results suggest that an attribute-based model should allow consumer preferences to vary among the constituent attributes. According to the empirical evidence presented here, consumers may present different state-dependence patterns for every attribute dependent upon the consumption history.

The empirical results also reveal significant non-linear structures for the partial utilities, and put forward that models should also be able to capture mixtures of inertia and variety seeking as a more complex state-dependence pattern. From a consumer-choice perspective, the major predictions of our theoretical framework are confirmed by the empirical illustration:

- The hybrid formulation has a superior fit compared to a linear state-dependence formulation.

- For every market studied, there are relevant hybrid segments presenting negative and significant non-linear coefficients. The estimated utility functions support the theoretical assumptions that consumers behave strongly inertial when the attributes are new, or their stock levels are low. This trend may be reversed and consumers may seek variety once the attribute has been accumulated continuously for several consumption periods.

Important managerial implications of this research can be emphasized. The results reveal the importance of modeling and estimating the different sources of loyalty presented in the purchasing patterns to understand consumers' preferences. In order to develop effective promotional strategies, firms need to know the preference drivers across segments. An inertial purchasing pattern for a brand may be a result of a preferred combination of attributes. If this is the case, consumers are loyal to the preferred brand, and the promotional efforts run by competitive products will only have a short-term effect. However, loyal purchasing patterns may also happen just because the purchased items contain attribute categories of a high level of familiarity for an inertial consumer. In this case, consumers simply do not switch to a competitive product because they are unfamiliar with its compounding attributes. For these inertial consumers, continuity promotional strategies aimed at increasing the level of familiarity for a competing product may have a long-term effect, as preferences are mainly driven by familiarity.

From a managerial perspective, the model offers a useful radiography of the competing products available in the market place for the category studied. Unlike the alternative-specific choice models, estimating the attribute-specific preference structure provides meaningful and 
interpretable parameters. For some frequently purchased categories, like yogurts or ice cream, consumers seek variety in flavors while behaving inertial for brands (see Kim et al. 2002). This behavioral pattern has relevant managerial implications, as an appropriate line extension for the brand is needed to guarantee the required switching by consumers across the available product varieties while being brand loyal. The model may constitute a decision-making tool enabling estimation of the impact of several marketing strategies across segments to be evaluated, such as in relation to the launch of new products or possibly extending existing lines. The results of the hybrid model can also have implications for pricing and promotional activities, such as cross-promotional offers (e.g. for which groups of products and for which segments will a joint promotion be more effective, depending on the variety-seeking levels shown for the implied combinations of attributes). 


\section{References}

Bawa, Kapil (1990), "Modeling Inertia and Variety Seeking Tendencies in Brand Choice Behavior," Marketing Science, 9 (Summer), 263-278.

Berlyne, D. C. (1963), "Motivational Problems Raised by Exploratory Behavior," in Psychology: A Study of a Science, (Ed.) S. Koch, New York: McGraw-Hill.

Berlyne, D.C. (1970), "Novelty, Complexity and Hedonic Value," Perception and Psychophysics, $8(5 \mathrm{~A}), 279-286$.

Bucklin, R. E., \& Gupta, S. (1992), "Brand Choice, Purchase Incidence, and Segmentation: An Integrated Approach," Journal of Marketing Research, 29 (May), 201-215.

Chintagunta, P. K. (1992), "Heterogeneity in Nested Logit Models: An Estimation Approach and Empirical Results," International Journal of Research in Marketing, 9, 161-175.

Dawes, Robin M. and B. Corrigan (1974), "Linear Models in Decision Making," Psychological Bulletin, 81 (2) 95-106.

Fader, Peter S. and Bruce G. S. Hardie (1996), "Modeling Consumer Choice Among SKU's," Journal of Marketing Research, 23 (November), 442-452.

Givon, Moshe M. (1984), "Variety Seeking Through Brand Switching," Marketing Science, 3 (Winter), 1-22.

Green, Paul E. and V. Srinivasan (1978), "Conjoint Analysis in Consumer Research: Issues and Outlook," Journal of Consumer Research, 5 (September), 103-123.

Guadagni, Peter M. and John D.C. Little (1983), "A Logit Model of Brand Choice Calibrated on Scanner Data," Marketing Science, 2 (Summer), 203-238.

Hardie, Bruce G. S., Eric J. Johnson and Peter S. Fader (1993), "Modeling Loss Aversion and Reference Dependence Effects on Brand Choice," Marketing Science, 12 (Fall), 378-394.

Jain, D. C., Vilcassim, N. J., \& Chintagunta, P. K. (1994), ”A Random-Coefficients Logit Brand-Choice Model Applied to Panel Data," Journal of Business 83 Economic Statistics, 12(3), 317-328.

Jeuland, Abel P. (1979), " Brand Choice Inertia as One Aspect of the Notion of Brand Loyalty," Management Science, 25 (July), 671-682. 
Jimenez-Martin, S., and A. Ladrón-de-Guevara (2007), "Modeling Consumption Patterns in the attribute space: theory and evidence of Hybrid Behavior," International Journal of Research in Marketing, 24 (3), 242-253.

Johnson, Eric J., Robert J. Meyer and Sanjoy Ghose (1989), "When Choice Models Fail: Compensatory Models in Negatively Correlated Environments," Journal of Marketing Research, 26 (August), 255-270.

Kahn, Barbara E., Manohar U. Kalwani and Donald G. Morrison (1986), "Measuring VarietySeeking and Reinforcement Behaviors Using Panel Data," Journal of Marketing Research, 23, $89-100$.

Kamakura, W.A., \& Russell, G. J. (1989), "A Probabilistic Choice Model for Market Segmentation and Elasticity Structure," Journal of Marketing Research, 26, 379-390.

Keane, M. (1997), "Modeling Heterogeneity and State Dependence in Consumer Choice Behavior.” Journal of Business Economic Statistics, 15, No 3 (July), 310-327.

Kim, J., Allenby G. M., \& Rossi, P. E. (2002), "Modeling Consumer Demand for Variety." Marketing Science, 21, No 3 (Summer), 229-250.

Kuehn, A.A. (1962), "Consumer Brand Choice - A Learning Process?," Journal of Advertising Research, 2, 10-17.

Lancaster, Kelvin (1971), Consumer Demand, A New Approach, New York: Columbia University Press.

Lattin, James M. (1987), "A Model of Balanced Choice Behavior," Marketing Science, 6 (Winter), 48-65.

Lattin, James M. and Leigh McAlister (1985), "Using A Variety-Seeking Model to Identify Substitute and Complementary Relationships Among Competing Products," Journal of Marketing Research, 22, 330-339.

McAlister Leigh (1982), "A Dynamic Attribute Satiation Model of Variety Seeking Behavior," Journal of Consumer Research, 9 (September), 141-150.

McAlister, Leigh and Edgar A. Pessemier (1982), "Variety Seeking Behavior: An InterDisciplinary Review," Journal of Consumer Research, 9 (December), 311-322. 
McFadden, D. (1974) "Conditional Logit Analysis of Qualitative Choice," in P. Zarembka (Ed.), Frontiers in Econometrics, New York: Academic Press.

Morrison, D. (1966), "Testing Brand-Switching Models," Journal of Marketing Research, 3 (November), 401-409.

Roy, R., Chintagunta, P. K., \& Haldar, S. (1996), "A Framework for Investigating Habits, "The Hand of the Past," and Heterogeneity in Dynamic Brand Choice," Marketing Science 15, No 3, 280-299.

Thaler, Richard (1985), "Mental Accounting and Consumer Choice," Marketing Science, 4 (Summer), 199-214.

Tversky, A. and D. Kahneman (1991), "Loss Aversion and Riskless Choice: A Reference Dependent Model," Quarterly Journal of Economics, 106 (November), 1039-1061.

Wierenga, B. (1974), An Investigation of Brand Choice Processes, Rotterdam: Universitaire Pers Rotterdam. 